\title{
Promoting effect of rapamycin on osteogenic differentiation of maxillary sinus membrane stem cells
}

\author{
Yanjun Lin ${ }^{1}$, Min Zhang ${ }^{2}$, Lin Zhou ${ }^{3}$, Xuxi Chen ${ }^{3}$, Jiang Chen ${ }^{3}$, Dong Wu ${ }^{\text {Corresp. } 3}$ \\ ${ }^{1}$ Fujian Key Laboratory of Oral Diseases, Fujian Medical University, Fuzhou, Fujian, China \\ 2 Fujian Provincial Engineering Research Center of Oral Biomaterial, Fujian Medical University, Fuzhou, Fujian, China \\ 3 Research Center of Dental and Craniofacial Implants, Fujian Medical University, Fuzhou, Fujian, China \\ Corresponding Author: Dong Wu \\ Email address: wudong@fjmu.edu.cn
}

Background: Stem cells located in the maxillary sinus membrane can differentiate into osteocytes. Our study aimed to evaluate the effect of rapamycin (RAPA) on the osteogenic differentiation of maxillary sinus membrane stem cells (MSMSCs). Methods: Colonyforming unit assay, immunophenotype identification assay, and multi-differentiation assay confirmed characteristics of MSMSCs obtained from SD rats. Transmission electron microscopy (TEM) and flow cytometry (FCM) identified the initial autophagic level of MSMSCs induced by RAPA. Real-time quantitative PCR (qPCR) evaluated subsequent autophagic levels and osteogenic differentiation. Alkaline phosphatase (ALP) activity assay and alizarin red staining (ARS) evaluated subsequent osteogenic differentiation. We performed a histological examination to clarify in vivo osteogenesis with ectopic bone mass from BALB/c nude mice. Results: MSMSCs possessed an active proliferation and multi-differentiation capacity, showing a phenotype of mesenchymal stem cells. The autophagic level increased with increasing $\operatorname{RAPA}(0,10,100,1000 \mathrm{nM})$ and decreased over time. ALP activity and calcium nodules forming in four RAPA-treated groups on three-time points $(7,14,21$ d) showed significant differences. Col1a1, Runx2, and Spp1 expressed most in $100 \mathrm{nM}$ RAPA group on 7 and $14 \mathrm{~d}$. Osteogenesis-related genes except for Ibsp expression between four groups tended to be consistent on $21 \mathrm{~d}$. $100 \mathrm{nM}$ and $10 \mathrm{nM}$ RAPAtreated groups showed more bone formation in vivo. Conclusion: RAPA can promote osteogenic differentiation of MSMSCs, indicating a possible relationship between osteogenic differentiation and autophagy. 


\title{
2 Promoting effect of rapamycin on osteogenic
}

3 differentiation of maxillary sinus membrane stem cells

4

5

6

7

8

\author{
Yan-Jun Lin1†, Min Zhang2, Lin Zhou3, Xu-Xi Chen3, Jiang Chen3, Dong Wu3*
}

1 Fujian Key Laboratory of Oral Diseases, Fujian Medical University, Fuzhou, Fujian, China

2 Fujian Provincial Engineering Research Center of Oral Biomaterial, Fujian Medical University, Fuzhou, Fujian, China

3 Research Center of Dental and Craniofacial Implants, Fujian Medical University, Fuzhou, Fujian, China

Corresponding Author:

Dong Wu1

No. 246, Yangqiao Road, Gulou District, Fuzhou, Fujian 350002, China

Email address: wudong@,fjmu.edu.cn

\section{Abstract}

Background: Stem cells located in the maxillary sinus membrane can differentiate into osteocytes. Our study aimed to evaluate the effect of rapamycin (RAPA) on the osteogenic differentiation of maxillary sinus membrane stem cells (MSMSCs).

Methods: Colony-forming unit assay, immunophenotype identification assay, and multidifferentiation assay confirmed characteristics of MSMSCs obtained from SD rats. Transmission electron microscopy (TEM) and flow cytometry (FCM) identified the initial autophagic level of MSMSCs induced by RAPA. Real-time quantitative PCR (qPCR) evaluated subsequent autophagic levels and osteogenic differentiation. Alkaline phosphatase (ALP) activity assay and alizarin red staining (ARS) evaluated subsequent osteogenic differentiation. We performed a histological examination to clarify in vivo osteogenesis with ectopic bone mass from BALB/C nude mice.

Results: MSMSCs possessed an active proliferation and multi-differentiation capacity, showing a phenotype of mesenchymal stem cells. The autophagic level increased with increasing RAPA $(0,10,100,1000 \mathrm{nM})$ and decreased over time. ALP activity and calcium nodules forming in four RAPA-treated groups on three-time points $(7,14,21 \mathrm{~d})$ showed significant differences. Col1a1, Runx2, and Spp1 expressed most in 100 nM RAPA group on 7 and $14 \mathrm{~d}$. Osteogenesis-related genes except for Ibsp expression between four groups tended to be consistent on $21 \mathrm{~d} .100 \mathrm{nM}$ and $10 \mathrm{nM}$ RAPA-treated groups showed more bone formation in vivo.

Conclusion: RAPA can promote osteogenic differentiation of MSMSCs, indicating a possible relationship between osteogenic differentiation and autophagy. 


\section{Introduction}

43 Maxillary sinus membrane elevation is a frequent option when bone height in the posterior

44

45

46

47

48

49

50

51

52

53

54

55

56

57

58

59

60

61

62

63

64

65

66

67

68

69

70

71

72

73

74

75

76

77

78

79

80

81

maxilla is less than $4 \mathrm{~mm}$. Maxillary sinus membrane elevation consists of two situations, bone augmentation with or without bone substitutes. Our previous research observed satisfying alveolar bone gains and a nearly $100 \%$ survival rate of implants using a maxillary sinus membrane elevation technique (Chen et al., 2016). When we elevate the maxillary sinus membrane without bone substitutes, there is still new bone formation underneath the membrane, even though it is less than that when elevating with bone substitutes (Yan et al., 2018).

For the time being, there are some interpretations of new bone formation underneath the Schneiderian membrane after surgery. Bone marrow stromal cells (BMSCs) immigrating from - jaw marrow stroma to its surface can produce new bone, which may be the primary cell source (Chen et al., 2018). Meanwhile, blood with productive growth factors can provide scaffolds and activators (Bonazza et al., 2018). Additionally, maxillary sinus membrane stem cells (MSMSCs) feature in the formation of new bone underneath the maxillary sinus membrane (Berbéri et al., 2017; Srouji et al., 2010). The maxillary sinus membrane is different from the periosteum. It consists of four layers (pseudostratified ciliated columnar epithelium, basement membrane, lamina propria, and periosteum-like structure) (Srouji et al., 2009). MSMSCs from the periosteum-like layer can differentiate into an osteogenic lineage and express osteogenic markers such as ALP, RUNX2, OCN, ON, and BSP (Guo et al., 2015). MSMSCs, similar to other mesenchymal stem cells (MSCs), are a reliable source of bone formation. Regardless of some studies on MSMSCs, examples of applications are rare, and the possible mechanisms of osteogenic differentiation remain unknown. In a previous study, MSCs showed a high level of basal autophagy that decreased during differentiation (Oliver et al., 2012). An additional study indicated that the maintenance of cellular stemness and the lineage determination of MSCs were deeply affected by autophagy (Sbrana et al., 2016). Rapamycin (RAPA) is a specific mTOR inhibitor that activates autophagy and promotes osteogenic differentiation of BMSCs (Wan et al., 2017).

Several papers reported that autophagy drove osteogenic differentiation of mesenchymal stem cells. (Pantovic et al., 2013; Vidoni et al., 2019; Wan et al., 2017). Up to date, no study has focused on the relationship between autophagy and osteogenic differentiation of MSMSCs.

Moreover, no study has focused on the effect of RAPA on MSMSCs. Therefore, the effect of RAPA on osteogenic differentiation of MSMSCs is an issue to investigate.

This study aimed to delve into the effect of RAPA on the osteogenic differentiation of MSMSCs. Colony-forming unit (CFU) assay, multi- differentiation assay, and immunophenotype identification assay evaluated cellular stemness. The autophagic level was validated by transmission electron microscopy (TEM) and flow cytometry (FCM). Real-time quantitative PCR (RT-qPCR) monitored autophagic markers (Becn1, LC3). Bone formation ex vivo was evaluated by alkaline phosphatase (ALP) activity assay and alizarin red staining (ARS). Furthermore, RT-qPCR monitored osteogenic markers (Colla1, Ibsp, Runx2, Spp1). Ectopic bone formation assay evaluated bone formation in vivo.

PeerJ reviewing PDF | (2020:06:49677:2:1:NEW 29 Apr 2021) 


\section{Materials \& Methods}

\section{Animals}

85 A total of SD male rats $(\mathrm{N}=50)$ and $\mathrm{BALB} / \mathrm{c}$ male nude mice $(\mathrm{N}=20)$ (SLAC Laboratory Animal

86 Co., Ltd., Shanghai, China) were housed in the Department of Comparative Medicine, the 900th

87 Hospital of Joint Logistics Support Force of the Chinese People's Liberation Army. No more

88 than five animals per cage were in pathogen-free and sterilized space with Sani-chip bedding and

89 a standard light-dark cycle. The environment temperature was $23 \pm 2^{\circ} \mathrm{C}$, and the humidity was 50 -

$9060 \%$ with proper ventilation. According to rodent standard feeding rules, the animals were fed

91 with standard laboratory tap water and commercial purified diets. SD rats were sacrificed using

92 ten times the dose of conventional anesthesia with pentobarbital ( 1\% sodium pentobarbital, 40

$93 \mathrm{mg} / \mathrm{kg}$ ) by intraperitoneal injection to obtain maxillary sinus membrane cells. We established an

94 ectopic subcutaneous osteogenesis model using BALB/c nude mice. BALB/c nude mice were

95 euthanized with pentobarbital ( $1 \%$ sodium pentobarbital, $50 \mathrm{mg} / \mathrm{kg}$ ) by intraperitoneal injection

96 after one month of feeding to obtain the specimen. To induce loss of consciousness and death

97 with a minimum of pain and distress, they were euthanized with overdose pentobarbital (10

98 times of general anesthesia) after surgery. Rigor mortis confirmed the death. After obtaining the

99 specimen, the animal carcasses were collectively processed by the animal center. Criteria

100 established for euthanizing animals before the planned end of the experiment followed AVMA

101 guidelines. All animal procedures followed the NIH guide of Humane Use and Care Animals and

102 were approved by the Institutional Ethics Committee of the School of Stomatology, Fujian

103 Medical University (Ref. [2017] NO.59).

104

105

106

\section{Cell culture}

107

108

Maxillary sinus membrane samples were separated carefully from SD rats. The tissues were cut into pieces and digested in a solution composed of $3 \mathrm{mg} / \mathrm{mL}$ collagenase type I and $4 \mathrm{mg} / \mathrm{mL}$ dispase (Sigma, USA) for 50 minutes at $37^{\circ} \mathrm{C}$. Samples were pooled and filtered by a $70 \mu \mathrm{m}$ strainer (Falcon, USA) to obtain single-cell suspensions. Single-cell suspensions $\left(1 \times 10^{4}\right.$ cells $)$ were seeded into $75 \mathrm{~cm}^{2}$ culture dishes (Corning, USA) with alpha modification of Eagle's medium (HyClone, USA) supplemented with 15\% fetal bovine serum(GIBCO, USA), $100 \mathrm{U} / \mathrm{ml}$ penicillin, and $100 \mathrm{mg} / \mathrm{ml}$ streptomycin (HyClone, USA). Suspensions were incubated at $37^{\circ} \mathrm{C}$ in $5 \% \mathrm{CO}_{2}$. Once cells reached $80 \%$ confluence, they were passaged with a ratio of $1: 3$. The cells at passage three were used in the following assays. Before osteogenic differentiation induction, cells were pretreated with RAPA $(0,10,100,1000 \mathrm{nM})$ for four hours.

116

117 CFU assay

$1181 \times 10^{3}$ MSMSCs at passage 3 were cultured for seven days in $60 \mathrm{~cm}^{2}$ dishes and stained with

$1190.1 \%$ crystal violet. Aggregates of $\geqslant 50$ cells were scored as colonies to assess colony-forming 120 efficiency. 


\section{Multi-differentiation assay}

123 Differentiation media kits (Cyagen, China) were used to induce osteogenic, adipogenic, and

124 chondrogenic differentiation. Respectively, cells at densities of $1 \times 10^{4} \mathrm{cell} / \mathrm{s} / \mathrm{cm}^{2}$ (osteogenic

125 induction), $3.2 \times 10^{4}$ cells $/ \mathrm{cm}^{2}$ (adipogenic induction) or $5 \times 10^{5} \mathrm{cells} / \mathrm{cm}^{2}$ (chondrogenic

126 induction) were seeded. Cells were fixed in 4\% paraformaldehyde for 15 minutes after $28 \mathrm{~d}$

127 (osteogenic induction), $16 \mathrm{~d}$ (adipogenic induction), and $21 \mathrm{~d}$ (chondrogenic induction).

128 Osteocytes were stained for 5 minutes with an alizarin red solution ( $\mathrm{pH} 4.2)$. Adipocytes were

129 stained for 15 minutes with oil red O. Chondrocytes were stained for 15 minutes with alcian

130 blue.

131

132

133

\section{Immunophenotype identification assay}

$1 \times 10^{6}$ MSMSCs at passage 3 were harvested and suspended in $500 \mu \mathrm{l}$ of PBS containing 20

134 $\mathrm{ng} / \mathrm{ml}$ AlexaFluor ${ }^{\circledR} 488$-coupled antibodies against CD34, CD45, CD90, CD146, and CD166. After incubation for 30 minutes at $4^{\circ} \mathrm{C}$, the cells were washed twice in PBS and analyzed by flow cytometry using the BD Accuri C6.

137

138

\section{Autophagy TEM analysis}

139

We treated the passage 3 MSMSCs seeded on glass slides with different RAPA $(0,10,100$, and $1000 \mathrm{nM}$ ) for four hours. Cells were fixed in $2.5 \%$ glutaraldehyde for 2 hours at $4^{\circ} \mathrm{C}$ and washed in phosphate-buffered saline (PBS). Cells were then fixed with $1 \% \mathrm{~K}_{2}\left[\mathrm{OsO}_{2}(\mathrm{OH})_{4}\right] \cdot 0.1 \mathrm{M} \mathrm{PBS}$ ( $\mathrm{pH}$ 7.4) for 2 hours. Sections $(70 \mathrm{~nm})$ were imaged on a transmission electron microscope (Tecnai G2 F20 S-TWIN, FEI, USA), and we captured autophagosomes as described in the results.

145

\section{Autophagy FCM analysis}

147 We treated the passage 3 MSMSCs with four groups of RAPA $(0,10,100$, and $1000 \mathrm{nM})$ for four hours. $1 \times 10^{6}$ cells were suspended in $500 \mu$ of PBS containing $50 \mathrm{nM}$ monodansylcadaverine (MDC). After incubation for 30 minutes at $4{ }^{\circ} \mathrm{C}$, the cells were washed with PBS and resuspended in $1 \mathrm{ml}$ of PBS for analysis using the BD Accuri C6.

\section{ALP activity assay}

The passage 3 MSMSCs at densities of $2 \times 10^{4}$ cells/well were seeded into 96-well plates (Corning, USA) and treated with four groups of RAPA (0, 10, 100, and $1000 \mathrm{nM})$ for four hours. According to the manufacturer's instructions, after 7, 14, and 21 days of culture in the calcification medium, ALP activity was detected using an ALP assay kit (Beyotime, China). The amount of ALP in the cells was normalized against the total protein content.

158

\section{Quantitative ARS assay}

160 The passage 3 MSMSCs at densities of $1 \times 10^{4}$ cells $/ \mathrm{cm}^{2}$ were seeded into 6-well plates 161 (Corning, USA) and treated with four groups of RAPA (0, 10, 100, and $1000 \mathrm{nM})$ for four hours. 
162 After 7, 14, and 21 days of culture in calcification medium, cells were fixed in 4\%

163

164

165

166

167

168

169

170

171

172

173

174

175

176

177

178

179

180

181

182

183

184

185

186

187

188

189

190

191

192

193

194

195

196

197

198

199

200

201

paraformaldehyde for 15 minutes and de-stained in $10 \mathrm{mmol} \mathrm{L}^{-1}$ sodium phosphate containing $10 \%$ cetylpyridinium chloride (Sigma, USA). The amount of alizarin red was quantified by the absorbance of the solution at $562 \mathrm{~nm}$ using a microplate reader.

\section{RT-qPCR}

According to the manufacturer's instructions, total RNA was isolated from passage 3 MSMSCs using Trizol reagent (TaKaRa, Japan). The purity and concentration of RNA were identified by UV spectroscopy. The Genomic DNA was excluded using gDNA Eraser in PrimeScript ${ }^{\mathrm{TM}}$ RT reagent Kit (TaKaRa, Japan) at $42{ }^{\circ} \mathrm{C}$ for $2 \mathrm{~min}$, then cDNA was synthesized with $1 \mu \mathrm{g}$ of total RNA. Real-time polymerase chain reaction assays were performed on triplicate samples using SYBR Premix Ex Taq ${ }^{\mathrm{TM}}$ II (Takara, Japan) in a Roche 480 Light Cycler (Roche, Mannheim, Germany). The cycling conditions consisted of incubating at $95^{\circ} \mathrm{C}$ for $30 \mathrm{~s}, 40$ cycles of $95^{\circ} \mathrm{C}$ for $5 \mathrm{~s}$, and $60^{\circ} \mathrm{C}$ for $30 \mathrm{~s}$. Gapdh as an internal control, we calculated relative expression levels of indicated genes by the $2^{-\Delta \Delta C t}$ method. Primer sequences were as follows (Table 1):

\section{Ectopic bone formation assay}

MSMSCs at passage 3 pretreated with different concentrations of RAPA were harvested and mixed with bone substitute materials (Bio-Oss ${ }^{\circledR}$, Geistlich, Switzerland). We transplanted the cell pellets into the flanks of nude mice. Four weeks after implantation, we terminated the mice, and harvested scaffolds for $\mathrm{H} \& \mathrm{E}$, Masson, and Goldner trichrome staining. Briefly, we cut the paraffin blocks into $4 \mathrm{~mm}$ sections; for H\&E staining, the sections were stained with Harris' hematoxylin and eosin for 2 minutes; for Masson staining, the sections were treated with a Masson staining fluid (Solarbio, China) for 5 minutes; for Goldner staining, the sections were treated with a Goldner staining fluid (Solarbio, China) for 5 minutes. We observed the results under a microscope.

\section{Statistical analysis}

Each experiment was performed in triplicate and repeated at least three times. We compared experimental groups using a two-way analysis of variance or repeated-measures ANOVA with SPSS 22.0 software. Values were presented as means \pm SD. All p-values were two-tailed, and $p$ $<0.05$ was considered statistically significant.

\section{Results}

\section{Characterization of MSMSCs}

Primary cells were successfully isolated from the SD rat maxillary sinus mucosa. They showed a spindle-shaped and fibroblast-like morphology, similar to BMSCs. MSMSCs possessed the active ability of proliferation and reached confluence after three days of culture in complete medium. $1 \times 10^{3}$ single cells were seeded at low density, forming $49 \pm 4.0$ colonies (1CFU-F per 204 cells) (Fig. 1A). Trilineage differentiation experiments validated the multi-differentiation 
202 potential of MSMSCs, including osteogenesis, adipogenesis, and chondrogenesis. After

203 osteogenic induction for 28 days, we observed calcium granules stained by Alizarin Red in 204 MSMSCs. After adipogenic induction for 14 days, we observed lipid droplets stained by Oil Red 205 O in MSMSCs. After chondrogenic induction for 14 days, we observed a sulfated proteoglycan206 rich matrix stained by Alcian blue in MSMSCs (Fig. 1B). Flow cytometry identified the 207 phenotype of MSMSCs. MSMSCs positively expressed MSC markers, CD90 and CD146, while 208 negatively expressing hematopoietic cell markers, CD34, and CD45. Otherwise, MSMSCs of SD 209 rats negatively expressed CD105 and CD166 (Fig. 1C).

210

211

212

213

214

215

216

217

218

219

220

221

222

223

224

225

226

227

228

229

230

231

232

233

234

235

236

237

238

239

240

241

\section{Validation of autophagy in MSMSCs}

Different concentrations of autophagy activator $(0 \mathrm{nM}, 10 \mathrm{nM}, 100 \mathrm{nM}$, and $1000 \mathrm{nM}$ RAPA) were used to pretreat MSMSCs for 4 hours prior to osteogenic differentiation. We observed autophagosomes by TEM (Fig. 2A). As the concentration increased, the number of autophagosomes in the cells gradually increased. Notably, in this study, MSMSCs treated with $1000 \mathrm{nM}$ RAPA showed the highest autophagosome concentrations. Interestingly, we found many amorphous organelles, but unexpectedly, these were at least partially encapsulated in autophagosomes. Additionally, the autophagosomes did not seem to be full of lysosomes. FCM detected autophagic vacuoles stained by MDC (Fig. 2B). Cells with $0 \mathrm{nM}$ RAPA were left untreated to probe for basal autophagosome recycling. The green fluorescence intensity of MSMSCs represented the autophagic level in the cell. The autophagic stimulated ratios were $1.33 \%$ of the $0 \mathrm{nM}$ RAPA-treated cells, $43.3 \%$ of the $10 \mathrm{nM}$ RAPA-treated, $78.9 \%$ of the 100 nM RAPA-treated and $98.0 \%$ of $1000 \mathrm{nM}$ RAPA-treated cells. The control group possessed a relatively low basal level of autophagy. Decuple RAPA concentration change was capable of notably altering the autophagic state of MSMSCs. Becnl and $L C 3$ expression represented subsequent levels of autophagy (Fig. 2C). The autophagic level after $7 \mathrm{~d}$ of osteogenic induction was still dose-dependent. The autophagic level was the highest in the $1000 \mathrm{nM}$ RAPA-treated group. However, over time in the osteogenic culture, the difference in Becnl and LC3 expression between the four groups became less.

\section{RAPA promoted ex vivo osteogenic differentiation of MSMSCs}

MSMSCs were induced to osteogenic differentiation for 7, 14, and 21 days after pretreatment with RAPA for 4 hours. BCIP/NBT staining represented the dynamic state of ALP. The outcomes showed that ALP expression in the four concentration groups was, in descending order, $100 \mathrm{nM}$ RAPA, $1000 \mathrm{nM}$ RAPA, $10 \mathrm{nM}$ RAPA, and $0 \mathrm{nM}$ RAPA. However, the difference between each group gradually decreased, developing with further differentiation. On the 21st day of osteogenic differentiation, the visible differences between the four groups were insignificant. ALP activity is an essential indicator of osteoblast differentiation. Therefore, we performed a quantitative ALP activity assay (Fig. 3A). ARS assessed calcium deposits. Calcium deposits increased and then reduced after pretreatment with RAPA (Fig. 3B). These changes were also dose-dependent. Real-time quantitative PCR was employed to detect the mRNA 
242 expression of Colla 1, Ibsp, Runx2, and Spp1 (Fig. 3C). The expression levels of osteogenesis243 related genes changed with RAPA treatment. This finding indicated that RAPA could alter the 244 development of osteogenic differentiation. In the early stage of osteogenic differentiation,

245 MSMSCs mainly expressed Runx2, and the Runx2 expression levels of the four groups gradually 246 achieved the same level, which indicated that the effect of RAPA on osteogenesis gradually 247 decreased. The change in expression of Collal was the same as Runx2 expression. Regarding 248 the expression of Sppl in the whole osteogenic stage, the 100 nM RAPA groups had the highest

249 Spp1 expression, and the $10 \mathrm{nM}$ RAPA and $1000 \mathrm{nM}$ RAPA groups had similar expression

250 levels. The expression of Ibsp, in the late stage of osteogenic differentiation, showed a difference

251

252

253

254

255

256

257

258

259

260

261

262

263

264

265

266

267

268

269

270

271

272

273

274

275

276

277

278

279

280

281 similar to that of OPN expression.

\section{RAPA promoted in vivo ectopic bone formation of MSMSCs}

To further examine the effect of autophagy on ectopic bone formation in vivo, we transplanted immunocompromised mice with MSMSCs pretreated with four concentrations of RAPA. Four weeks after transplantation, the bony masses that formed at the surgical sites were removed and analyzed. H \& E (Fig. 4A), Masson's trichrome (Fig. 4B), and Goldner's trichrome staining (Fig. 4C) revealed that RAPA might induce bone-like tissue formation in vivo. Similar to native bone tissue, the new bone-like structure contained osteocytes encased in the newly deposited bone matrix. We observed little bone formation in the RAPA-free groups. Interestingly, the numbers of new vessels formed in the $100 \mathrm{nM}$ and the $10 \mathrm{nM}$ RAPA-treated groups seemed more than that in the other two groups. It indicated that an appropriate RAPA treatment might have improved the new bone formation and improved the formation of new blood vessels, which created a virtuous circle of local bone formation.

\section{Discussion}

Osteogenic differentiation of MSMSCs was confirmed many years ago, indicating that the Schneiderian membrane possesses osteogenic potential (Guo et al., 2015). In this study, MSMSCs expressed MSCs relevant immunocytochemical markers and possessed the ability of osteogenic differentiation. Evidence shows that MSMSCs do not express CD105 and CD166 but do positively express CD90 and CD146. Reduced expression of CD90 can increase the level of osteogenic differentiation of MSCs. CD146 can be used to identify subpopulations of cells with osteogenic capacity in MSCs. We divided the osteogenic differentiation of MSCs into four stages: immature osteoprogenitor cells, mature osteoprogenitor cells, pre-osteoblasts, and mature bone cells. In these four stages, multiple factors regulate MSCs, and Runx2 is a crucial factor. Knocking out Runx2 in animal models results in the absence of skeletal systems in experimental mice (Takarada et al., 2013). RAPA can induce transcriptional activation of osteogenic differentiation through increased GATA4 and Sox17 that modulate downstream Runx2 (Gambacurta et al., 2019). Also, some markers often express in the osteogenic differentiation of MSCs. For example, ALP does not express in immature osteoprogenitor cells, but ALP expression increases gradually with osteogenic differentiation (Srouji et al., 2010). Collal is not 
282

283

284

285

286

287

288

289

290

291

292

293

294

295

296

297

298

299

300

301

302

303

304

305

306

307

308

309

310

311

312

313

314

315

316

317

318

319

320

321

expressed merely in immature osteoprogenitor cells (Rasi et al., 2015). Ibsp expresses in all four stages, but the difference in expression is vast (Bouleftour et al., 2016). The specific performance of osteogenic differentiation is the enhancement of Alp, Colla1, Ibsp, Sppl, and Runx 2 gene expression. Therefore, in this study, MSMSCs were subjected to osteogenic induction after RAPA pretreatment, and then Colla1, Ibsp, Runx2, and Sppl were selected as the target genes. A variety of factors are involved in regulating autophagosome formation, and LC3 is a crucial marker of autophagy (Tanida, Ueno \& Kominami, 2008). Besides, BECN1 is also one of the critical proteins regulating autophagy and can form complexes with related proteins to induce autophagy and regulate autophagy levels (Kang et al., 2011). Autophagy refers to the formation of autophagosomes by forming a membrane structure that encapsulates part of the cytoplasm, intracellular bodies, and proteins that need to degrade due to damage and senescence. Autophagy lysosomes achieve the physiological processes of cell homeostasis and organelle renewal by degrading their encapsulation (Mizushima \& Komatsu, 2011). This process also plays a role in the fate decision of MSCs (Green \& Levine, 2014). Osteogenic differentiation is a prevailing differentiation direction of MSCs, and studies have shown that autophagy plays a regulatory role in this process (Pantovic et al., 2013). The occurrence of autophagy is affected by many factors, and cells can increase the level of autophagy following induction by factors such as hypoxia, stress, and drug treatment (Ravanan, Srikumar \& Talwa, 2017). In the ex vivo experiments modeling autophagy, drug induction is the most common method for changing autophagy. Among them, autophagy agonists such as RAPA (Vizza et al., 2018) and Torin (Wang et al., 2015) and autophagy inhibitors such as 3-MA (Wang, Li, Chen, 2018) and Bafilomycin A1 (Wang et al., 2017) are conventional drug induction methods. As a classical mTOR inhibitor, RAPA can effectively induce stem cells to increase their autophagy levels in a dose-dependent manner (Sotthibundhu et al., 2016). RAPA induces autophagy by binding the mammalian RAPA target protein (mammalian target of RAPA, mTOR), causing mTOR signaling pathway cascade molecules to become active such that cells translate autophagy-related proteins (Wang \& Zhang, 2019). The dynamic observation of the number and morphology of autophagosomes under electron microscopy is the gold standard for autophagy detection. The number of autophagosomes in cells represents the level of autophagy to some extent (Soto-Burgos et al., 2018). In this study, we observed the number and morphology of autophagosomes in cells by TEM. MDC dye is eosinophilic fluorescent staining that is a specific marker stain for detecting autophagosome formation (Gan et al., 2017). In this study, we observed autophagy levels qualitatively by microscope and quantitatively by FCM.

Multiple factors regulate mesenchymal stem cell fate decisions. For example, the differentiation of MSCs into osteoblasts depends on physical, chemical, and biological factors (Rodolfo, Di Bartolomeo \& Cecconi, 2016). The level of autophagy decreases when cells differentiate into bone-like cells. The inhibition of autophagy could affect the survival and differentiation of MSCs (Gómez-Puerto et al., 2016). By detecting changes in autophagic flow and expression of osteogenic markers, autophagy is implicated in the osteogenic differentiation of MSCs. The osteogenic potential reduces when autophagy activity increases in MSCs. Following 
322 pretreatment with the autophagy inhibitor 3-MA and the autophagic agonist RAPA, BMMSCs

323

324

325

326

327

328

329

330

331

332

333

334

335

336

337

338

339

340

341

342

343

344

345

346

347

348

349

350

351

352

353

354

355

356

357

358

359

360

361

were transplanted in nude mice, and in vivo analysis showed that the ectopic osteogenesis quality increased and that the bone-like tissue increased in the RAPA-treated group; the recovery of the 3-MA-treated osteogenesis ability was inhibited (Wan et al., 2017). Previous studies showed that RAPA promoted the osteoblastic differentiation of human embryonic stem cells by blocking the $\mathrm{PI} 3 \mathrm{~K} / \mathrm{AKT} / \mathrm{mTOR}$ pathway and stimulating the BMP/Smad pathway (Arianna et al., 2017; Lee et al., 2010). Also, there are recent reports of studies on the epigenetics of MSMSC osteogenic differentiation. Among them, lnc-NTF3-5 and hsa_circRNA_33287 can positively regulate osteogenic differentiation of MSMSCs (Peng et al., 2018; Peng et al., 2019), and miR-1827 can negatively regulate osteogenic differentiation of MSMSCs (Zhu et al., 2017). It seems that further study may be related to epigenetic regulation of autophagy on the osteogenic differentiation of MSMSCs.

A previous study showed that RAPA did not have an osteogenic effect on MSCs but inhibited osteogenic differentiation induced by dexamethasone (Isomoto et al., 2007). However, the concentration of RAPA in this paper was $0.01 \mathrm{nM}$. RAPA inhibited endogenous mTOR with IC50 values of $\sim 0.1 \mathrm{nM}$ in HEK293 cells (Edwards et al., 2007). Nevertheless, it does not mean that the effective concentration of RAPA for osteogenic differentiation is in such a low nanomolar range.

Overtime in the osteogenic culture, the difference in Becn 1 and $L C 3$ expression between the four groups became less. It indicated that the effect of RAPA on MSMSCs gradually decreased over time, and the autophagic level decreased with differentiation. In this study, we used a variety of osteogenic markers to detect osteogenic differentiation. However, the autophagic marker was insufficient to illustrate the inherent relationship between autophagy and osteogenic differentiation. Thus, in future studies, more autophagic markers would be adopted. Moreover, to identify the effect, methods of gene silencing and over-expression would be used.

A few limitations should also be noted in this study. First, we did not perform a dose-response assay, which led to a lack of justification for the concentrations of RAPA. Second, we did not provide a MSMSCs proliferation assay result after four concentrations of RAPA treatment. The potential cytostatic effects of RAPA might influence the osteogenic differentiation of MSMSCs. Third, we did not select good autophagic markers and tested them on the 7th, 14th, and 21 st day. The evidence above could not link the RAPA-mediated induction of autophagy with the proosteogenic response. Fourth, we did not semi-quantify the results of the in vivo ectopic bone formation. Some nude mice died in the duration of transplantation, which caused an insufficient sample size of biological repetitions.

According to this study, RAPA seems to be the right choice for the atrophic posterior maxilla. We might suggest adding an appropriate amount of RAPA to bone substitutes when doing maxillary sinus membrane elevation. Otherwise, RAPA modified bone substitutes may show a better ability to osteogenesis. Furthermore, some older adults may prevent atrophic posterior maxilla by taking oral medications of RAPA.

PeerJ reviewing PDF | (2020:06:49677:2:1:NEW 29 Apr 2021) 


\section{Conclusions}

363 In conclusion, the results indicated that RAPA promoted the osteogenic differentiation of

364

365

366

367

368

369

370

371

372

373

374

375

376

377

378

379

380

381

382

383

384

385

386

387

388

389

390

391

392

393

394

395

396

397

398

399

MSMSCs within a specific range. Autophagy might play an essential role in the osteogenic differentiation of MSMSCs. The findings of the study also provided new insight into the prevention and treatment of atrophic posterior maxilla.

\section{Acknowledgments}

Thanks for rats and mice involved in the experiments and the 900th Hospital of Joint Logistics Support Force platform of the Chinese People's Liberation Army. We express our gratitude to anonymous reviewers for providing insightful comments.

\section{References}

Arianna C, Eliana C, Flavio A, Marco R, Giacomo D, Manuel S, Elena B, Alessandra G. 2017. Rapid Rapamycin-Only Induced Osteogenic Differentiation of Blood-Derived Stem Cells and Their Adhesion to Natural and Artificial Scaffolds. Stem Cells International 2017:2976541. DOI 10.1155/2017/2976541

Berbéri A, Al-Nemer F, Hamade E, Noujeim Z, Badran B, Zibara K. 2017. Mesenchymal stem cells with osteogenic potential in human maxillary sinus membrane: an in vitro study. Clinical Oral Investigations 21(5):1599-1609 DOI 10.1007/s00784-016-1945-6

Bonazza V, Borsani E, Buffoli B, Parolini S, Inchingolo F, Rezzani R, Rodella LF. 2018. In vitro treatment with concentrated growth factors (CGF) and sodium orthosilicate positively affects cell renewal in three different human cell lines. Cell Biology International 42(3):353-364 DOI 10.1002/cbin.10908

Bouleftour W, Juignet L, Bouet G, Granito RN, Vanden-Bossche A, Laroche N, Aubin JE, Lafage-Proust MH, Vico L, Malaval L. 2016. The role of the SIBLING, Bone Sialoprotein in skeletal biology-Contribution of mouse experimental genetics. Matrix Biology 52-54:60-77 DOI 10.1016/j.matbio.2015.12.011

Chen X, Wang J, Yu L, Zhou J, Zheng D, Zhang B. 2018. Effect of concentrated growth factor (CGF) on the promotion of osteogenesis in bone marrow stromal cells (BMSC) in vivo. Scientific Reports 8(1):5876 DOI 10.1038/s41598-018-24364-5

Chen Y, Cai Z, Zheng D, Lin P, Cai Y, Hong S, Lai Y, Wu D. 2016. Inlay osteotome sinus floor elevation with concentrated growth factor application and simultaneous short implant placement in severely atrophic maxilla. Scientific Reports 6:27348 DOI 10.1038/srep27348 Edwards SR, Wandless TJ. 2007. The rapamycin-binding domain of the protein kinase mammalian target of rapamycin is a destabilizing domain. Journal of Biological Chemistry 282(18):13395-401 DOI 10.1074/jbc.M700498200 
400 Gambacurta A, Merlini G, Ruggiero C, Diedenhofen G, Battista N, Bari M, Balsamo M, 401 Piccirillo S, Valentini G, Mascetti G, Maccarrone M. 2019. Human osteogenic differentiation 402 in Space: proteomic and epigenetic clues to better understand osteoporosis. Scientific Reports 403 9(1):8343 DOI 10.1038/s41598-019-44593-6

404 Gan LS, Zeng LW, Li XR, Zhou CX, Li J. 2017. New homoisoflavonoid analogues protect 405 cells by regulating autophagy. Bioorganic \& Medicinal Chemistry Letters 27(6):1441-1445 DOI $406 \quad$ 10.1016/j.bmcl.2017.01.086

407 Gómez-Puerto MC, Verhagen LP, Braat AK, Lam EW, Coffer PJ, Lorenowicz MJ. 2016.

408 Activation of autophagy by FOXO3 regulates redox homeostasis during osteogenic

409 differentiation. Autophagy 12(10):1804-1816 DOI 10.1080/15548627.2016.1203484

410 Green DR, Levine B. 2014. To be or not to be? How selective autophagy and cell death govern

411 cell fate. Cell 157(1):65-75 DOI 10.1016/j.cell.2014.02.049

412 Guo J, Weng J, Rong Q, Zhang X, Zhu S, Huang D, Li X, Chen S. 2015. Investigation of

413 multipotent postnatal stem cells from human maxillary sinus membrane. Scientific Reports

414 5:11660 DOI 10.1038/srep11660

415 Isomoto S, Hattori K, Ohgushi H, Nakajima H, Tanaka Y, Takakura Y. 2007. Rapamycin as

416 an inhibitor of osteogenic differentiation in bone marrow-derived mesenchymal stem cells.

417 Journal of Orthopaedic Science 12(1):83-88 DOI 10.1007/s00776-006-1079-9

418 Kang R, Zeh HJ, Lotze MT, Tang D. 2011. The Beclin 1 network regulates autophagy and

419 apoptosis. Cell Death and Differentiation 18(4):571-580 DOI 10.1038/cdd.2010.191

420 Lee KW, Yook JY, Son MY, Kim MJ, Koo DB, Han YM, Cho YS. 2010. Rapamycin

421 promotes the osteoblastic differentiation of human embryonic stem cells by blocking the mTOR

422 pathway and stimulating the BMP/Smad pathway. Stem Cells and Development 19(4):557-68.

423 DOI 10.1089/scd.2009.0147

424 Mizushima N, Komatsu M. 2011. Autophagy: renovation of cells and tissues. Cell 147(4):728-

425741 DOI 10.1016/j.cell.2011.10.026

426 Oliver L, Hue E, Priault M, Vallette FM. 2012. Basal autophagy decreased during the

427 differentiation of human adult mesenchymal stem cells. Stem Cells and Development

428 21(15):2779-2788 DOI 10.1089/scd.2012.0124

429 Pantovic A, Krstic A, Janjetovic K, Kocic J, Harhaji-Trajkovic L, Bugarski D, Trajkovic

430 V. 2013. Coordinated time-dependent modulation of AMPK/Akt/mTOR signaling and

431 autophagy controls osteogenic differentiation of human mesenchymal stem cells. Bone

432 52(1):524-531 DOI 10.1016/j.bone.2012.10.024

433 Peng W, Zhu SX, Chen J, Wang J, Rong Q, Chen SL. 2019. Hsa_circRNA_33287 promotes

434 the osteogenic differentiation of maxillary sinus membrane stem cells via miR-214-3p/Runx3.

435 Biomedicine \& Pharmacotherapy 109:1709-1717 DOI 10.1016/j.biopha.2018.10.159

436 Peng W, Zhu SX, Wang J, Chen LL, Weng JQ, Chen SL. 2018. Lnc-NTF3-5 promotes

437 osteogenic differentiation of maxillary sinus membrane stem cells via sponging miR-93-3p.

438 Clinical Implant Dentistry and Related Research 20(2):110-121 DOI 10.1111/cid.12553 
439 Rasi Ghaemi S, Delalat B, Cetó X, Harding FJ, Tuke J, Voelcker NH. 2016. Synergistic

440 influence of collagen I and BMP 2 drives osteogenic differentiation of mesenchymal stem cells:

441 A cell microarray analysis. Acta Biomaterialia 34:41-52 DOI 10.1016/j.actbio.2015.07.027

442 Ravanan P, Srikumar IF, Talwar P. 2017. Autophagy: The spotlight for cellular stress

443 responses. Life Sciences 188:53-67 DOI 10.1016/j.lfs.2017.08.029

444 Rodolfo C, Di Bartolomeo S, Cecconi F. Autophagy in stem and progenitor cells. 2016.

445 Cellular and Molecular Life Sciences 73(3):475-496 DOI 10.1007/s00018-015-2071-3

446 Sbrana FV, Cortini M, Avnet S, Perut F, Columbaro M, De Milito A, Baldini N. 2016. The

447 role of autophagy in the maintenance of stemness and differentiation of mesenchymal stem cells.

448 Stem Cell Reviews and Reports 12(6):621-633 DOI 10.1007/s12015-016-9690-4

449 Soto-Burgos J, Zhuang X, Jiang L, Bassham DC. 2018. Dynamics of autophagosome

450 formation. Plant Physiology 176(1):219-229 DOI 10.1104/pp.17.01236

451 Sotthibundhu A, McDonagh K, von Kriegsheim A, Garcia-Munoz A, Klawiter A,

452 Thompson K, Chauhan KD, Krawczyk J, McInerney V, Dockery P, Devine MJ, Kunath T,

453 Barry F, O'Brien T, Shen S. 2016. Rapamycin regulates autophagy and cell adhesion in

454 induced pluripotent stem cells. Stem Cell Research \& Therapy 7(1):166 DOI 10.1186/s13287-

455 016-0425-x

456 Srouji S, Ben-David D, Lotan R, Riminucci M, Livne E, Bianco P. 2010. The innate

457 osteogenic potential of the maxillary sinus (Schneiderian) membrane: an ectopic tissue transplant

458 model simulating sinus lifting. International Journal of Oral and Maxillofacial Surgery

459 39(8):793-801 DOI 10.1016/j.ijom.2010.03.009

460 Srouji S, Kizhner T, Ben David D, Riminucci M, Bianco P, Livne E. 2009. The Schneiderian

461 membrane contains osteoprogenitor cells: in vivo and in vitro study. Calcified Tissue

462 International 84(2):138-145 DOI 10.1007/s00223-008-9202-x

463 Takarada T, Hinoi E, Nakazato R, Ochi H, Xu C, Tsuchikane A, Takeda S, Karsenty G,

464 Abe T. 2013. An analysis of skeletal development in osteoblast-specific and chondrocyte-

465 specific runt-related transcription factor-2 (Runx2) knockout mice. Journal of Bone and Mineral

466 Research 28(10):2064-2069 DOI 10.1002/jbmr.1945

467 Tanida I, Ueno T, Kominami E. 2008. LC3 and autophagy. Methods in Molecular Biology

468 445:77-88 DOI 10.1007/978-1-59745-157-4_4

469 Vidoni C, Ferraresi A, Secomandi E, Vallino L, Gardin C, Zavan B, Mortellaro C,

470 Isidoro C. 2019. Autophagy drives osteogenic differentiation of human gingival mesenchymal

471 stem cells. Cell Communication and Signaling 17(1):98 DOI 10.1186/s12964-019-0414-7

472 Vizza D, Perri A, Toteda G, Lupinacci S, Perrotta I, Lofaro D, Leone F, Gigliotti P, La

473 Russa A, Bonofiglio R. 2018. Rapamycin-induced autophagy protects proximal tubular renal

474 cells against proteinuric damage through the transcriptional activation of the nerve growth factor

475 receptor NGFR. Autophagy 14(6):1028-1042 DOI 10.1080/15548627.2018.1448740

476 Wan Y, Zhuo N, Li Y, Zhao W, Jiang D. 2017. Autophagy promotes osteogenic differentiation

477 of human bone marrow mesenchymal stem cell derived from osteoporotic vertebrae. 
478 Biochemical and Biophysical Research Communications 488(1):46-52 DOI

479 10.1016/j.bbrc.2017.05.004

480 Wang C, Wang X, Su Z, Fei H, Liu X, Pan Q. 2015. The novel mTOR inhibitor Torin-2

481 induces autophagy and downregulates the expression of UHRF1 to suppress hepatocarcinoma

482 cell growth. Oncology Reports 34(4):1708-1716 DOI 10.3892/or.2015.4146

483 Wang L, Li X, Chen C. 2018. 3-methyadenine attenuates chloroform-induced hepatotoxicity 484 via autophagy activation. BioMed Research 39(2):87-89 DOI 10.2220/biomedres.39.87

485 Wang Q, You T, Fan H, Wang Y, Chu T, Poncz M, Zhu L. 2017. Rapamycin and 486 bafilomycin A1 alter autophagy and megakaryopoiesis. Platelets 28(1):82-89 DOI $487 \quad 10.1080 / 09537104.2016 .1204436$

488 Wang Y, Zhang H. 2019. Regulation of autophagy by mTOR signaling pathway. Advances in 489 Experimental Medicine and Biology 1206:67-83 DOI 10.1007/978-981-15-0602-4_3

490 Yan M, Liu R, Bai S, Wang M, Xia H, Chen J. 2018. Transalveolar sinus floor lift without 491 bone grafting in atrophic maxilla: A meta-analysis. Scientific Reports 8(1):1451 DOI 492 10.1038/s41598-018-19515-7

493 Zhu S, Peng W, Li X, Weng J, Zhang X, Guo J, Huang D, Rong Q, Chen S. 2017. miR-1827

494 inhibits osteogenic differentiation by targeting IGF1 in MSMSCs. Scientific Reports 7:46136 495 DOI 10.1038/srep46136 


\section{Table $\mathbf{1}$ (on next page)}

Primer sequences for real-time RT-PCR 


\begin{tabular}{llll}
\hline \multicolumn{2}{l}{ Table1 Primer sequences for real-time RT-PCR } \\
\hline Gene & Access & Forward & Reverse \\
Gapdh & NM_017008 & GTATGACAATGAATATGGCTACAG & TCTCTTGCTCTCAGTATCCTTG \\
LC3 & NM_012823 & GGCTCTGGCTATTCTGTCTC & CTGACTTACATCTGGTGCTGAA \\
Becn1 & NM_001034117 & GCGGCTCCTATTCCATCAA & AGCATCTTTCCAAACCAAACAAA \\
Colla1 & NM_053304 & AAAGATGGACTCAACGGTCTC & CAGGAAGCTGAAGTCATAACCA \\
Ibsp & NM_012587 & AACCTTAGCCGTTCAGATGT & CAGACACCACTGTAACCTAGAA \\
Runx2 & NM_001278483 & GGACCGACACAGCCATAT & GGAAGGATGAGAGCCAACT \\
Spp1 & NM_012881 & CAGACACCACTGTAACCTAGAA & TTGCCTGCCTCTACATACATT \\
\hline
\end{tabular}




\section{Figure 1}

\section{Characterization of MSMSCs.}

(A) Representative image of a single colony-forming unit of MSMSCs. Scale bars represent $200 \mu \mathrm{m}$ and $80 \mu \mathrm{m}$, respectively. (B) Representative images of the osteogenic, adipogenic, and chondrogenic differentiation of MSMSCs. Scale bars represent $100 \mu \mathrm{m}$. (C) Cell surface markers of MSMSCs; representative figures of cytometric flow tests and percentage of positive expression. 
A
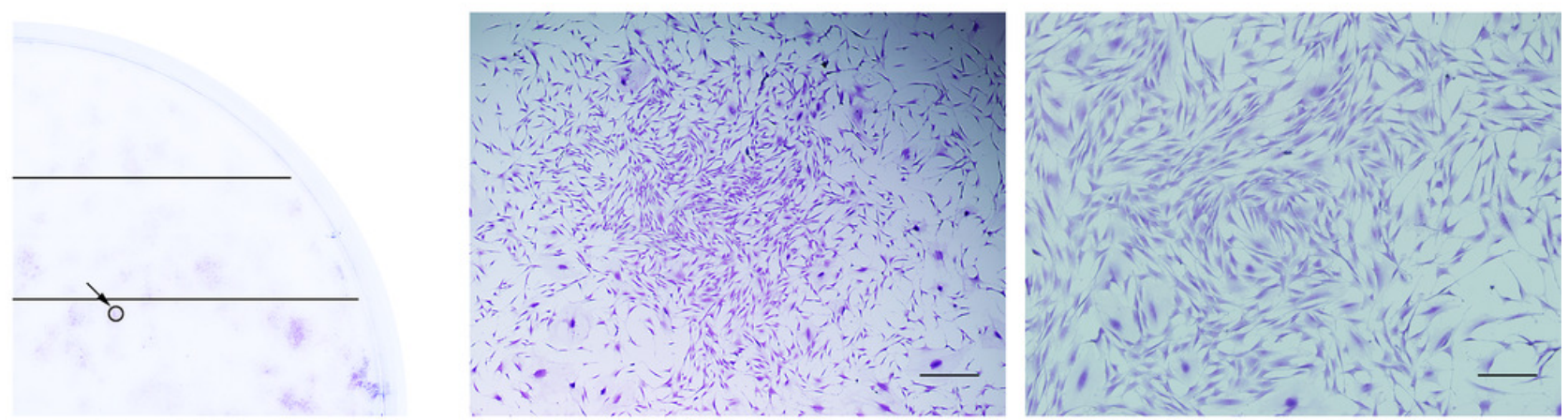

B
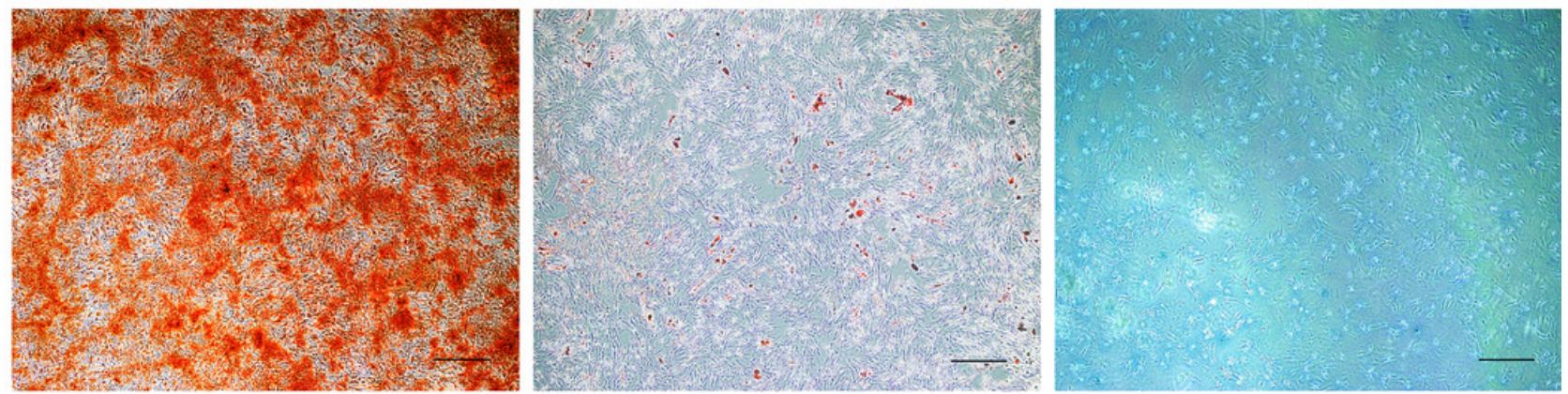

C
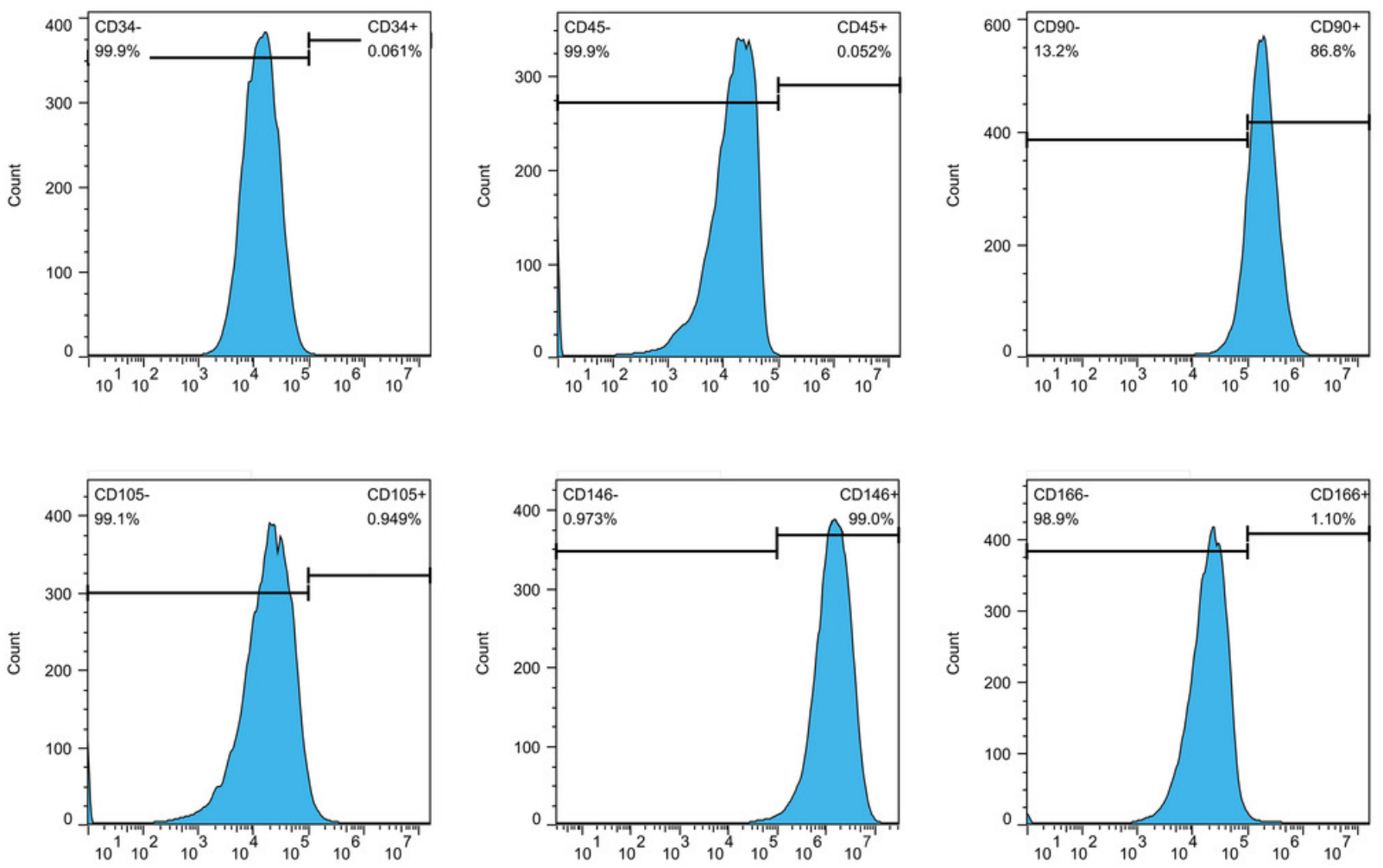

\begin{tabular}{cccccc}
\hline CD34 & CD45 & CD90 & CD105 & CD146 & CD166 \\
\hline $0.06 \%$ & $0.05 \%$ & $86.80 \%$ & $0.95 \%$ & $98.30 \%$ & $1.10 \%$ \\
\hline
\end{tabular}




\section{Figure 2}

Autophagic levels of MSMSCs after pretreatment with four concentrations of RAPA for 4 hours.

(A) Representative figures of transmission electron microscopy. Scale bars represent $1 \mu \mathrm{m}$.

(B) Representative figures of cytometric flow tests and percentage of positive expression. (C) The gene expression levels of the autophagic marker $L C 3$ were examined by qPCR. (D) The gene expression levels of the autophagic markers Becn1 were examined by qPCR. Relative mRNA expression was normalized to the control. The values are expressed as the mean \pm SD. $* p<0.05$. 

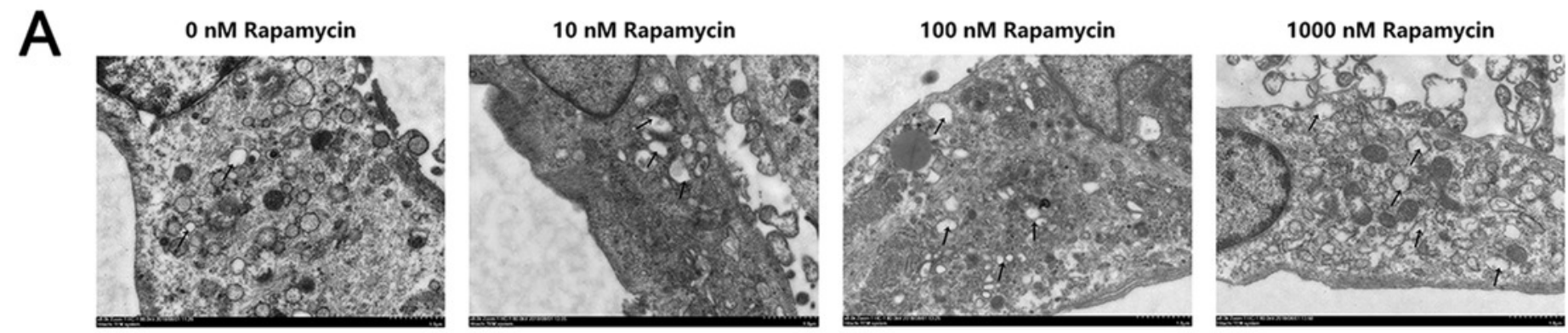

B
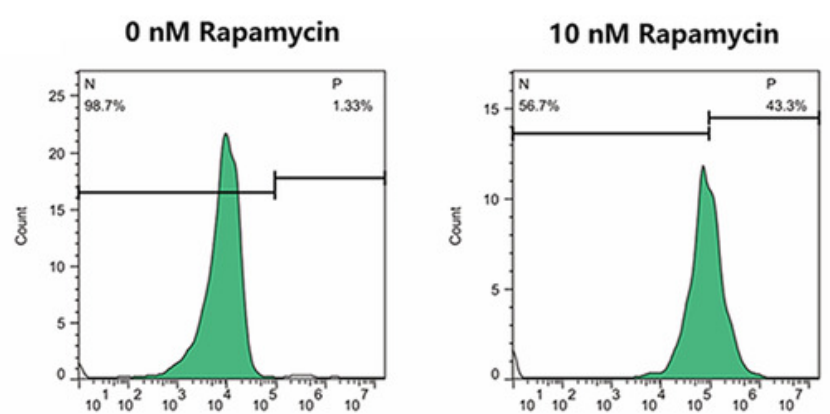

100 nM Rapamycin

1000 nM Rapamycin

RAPA (nM) 0

\begin{tabular}{lll}
10 & 100 & 1000 \\
\hline $43.30 \%$ & $78.90 \%$ & $98.00 \%$ \\
\hline
\end{tabular}
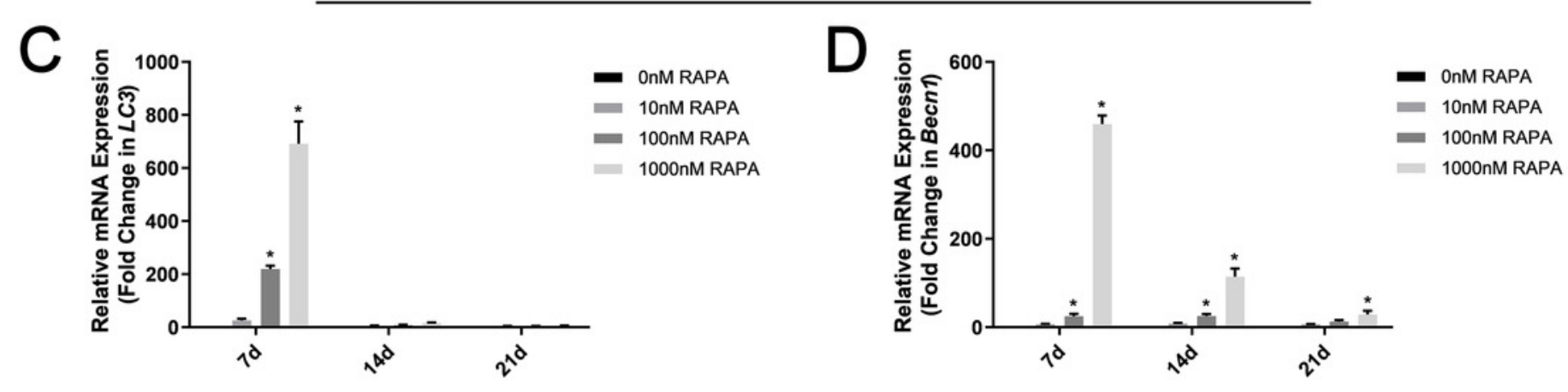


\section{Figure 3}

RAPA upregulated ex vivo osteogenic differentiation in MSMSCs.

(A) Representative images of four groups over three-time points using BCIP/NBT staining.

Scale bars represent $100 \mu \mathrm{m}$. (B) Representative images of the four groups over three-time points using Alizarin Red staining. Scale bars represent $100 \mu \mathrm{m}$. (C) ALP activities were detected by the alkaline phosphatase assay kit. (D) The total areas of mineralized nodules in the different groups were quantified using cetylpyridinium chloride. (E) The gene expression levels of the osteogenic marker Colla1 were examined by GPCR. (F) The gene expression levels of the osteogenic marker Ibsp were examined by qPCR. (G) The gene expression levels of the osteogenic marker Runx2 were examined by qPCR. (H) The gene expression levels of the osteogenic marker Spp1 were examined by qPCR. Relative mRNA expression was normalized to the control. All the values above are expressed as the mean \pm SD. ${ }^{*} p<0.05$. 


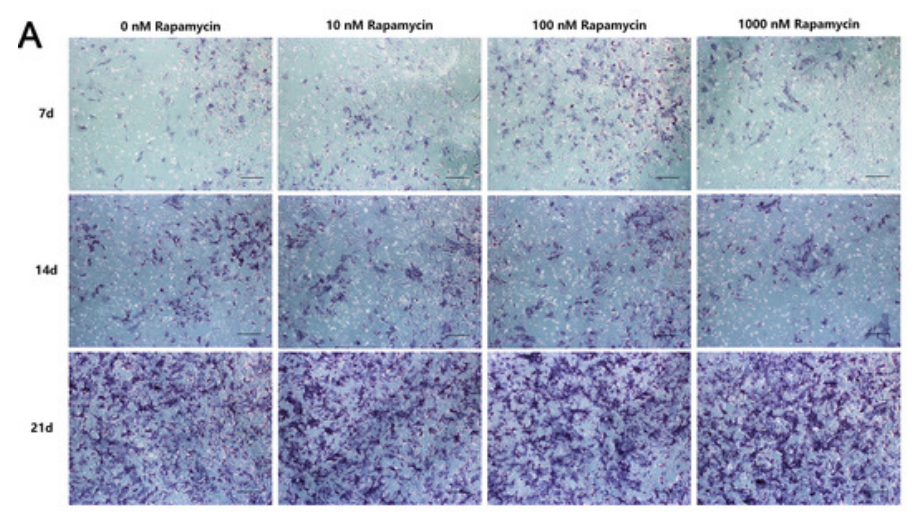

C

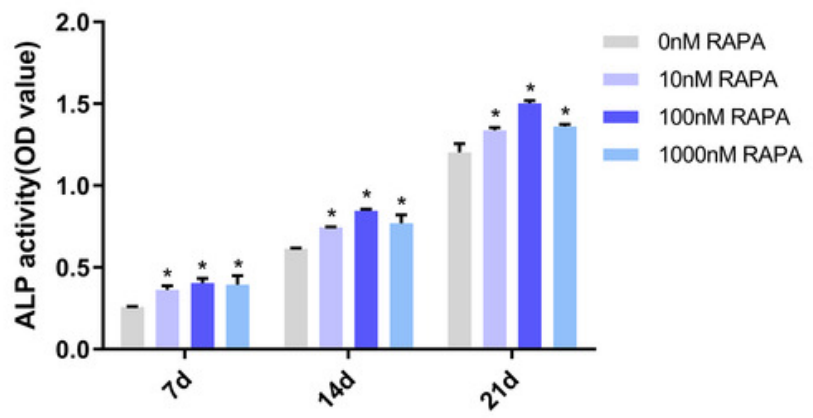

E

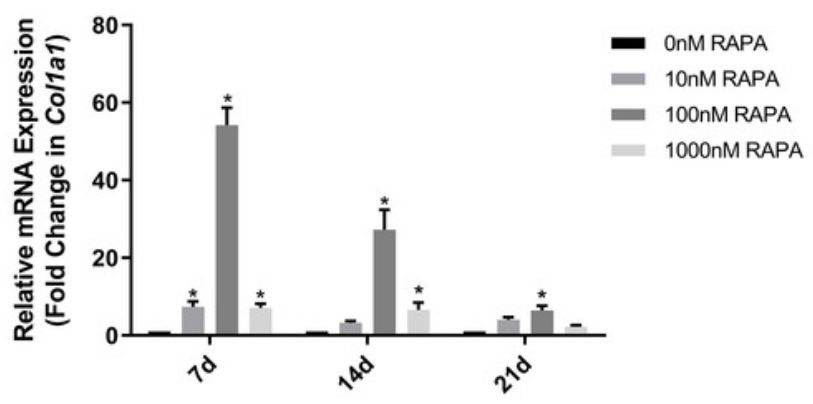

G

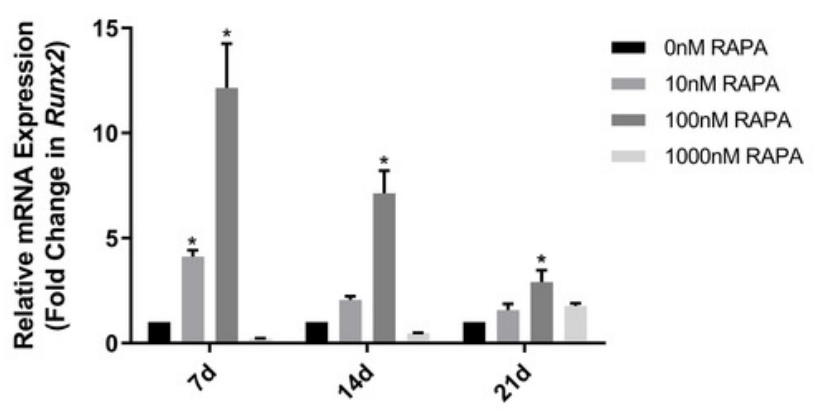

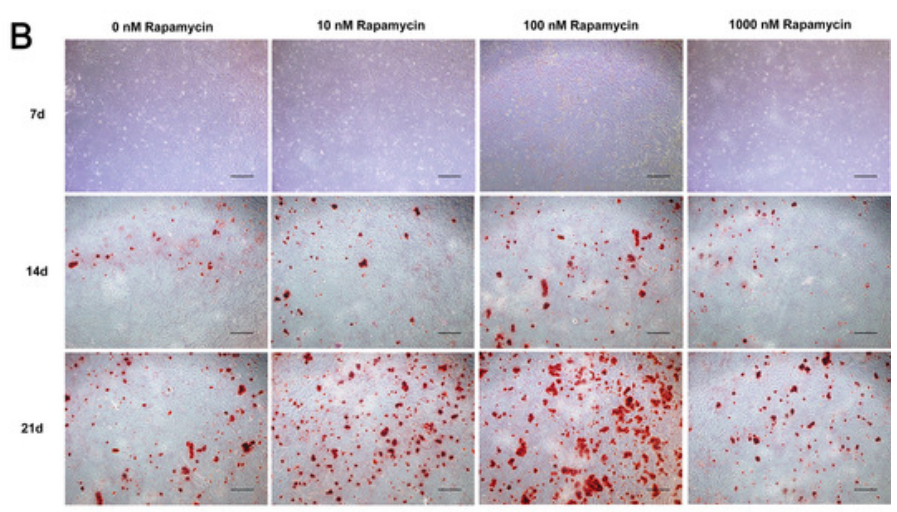

D

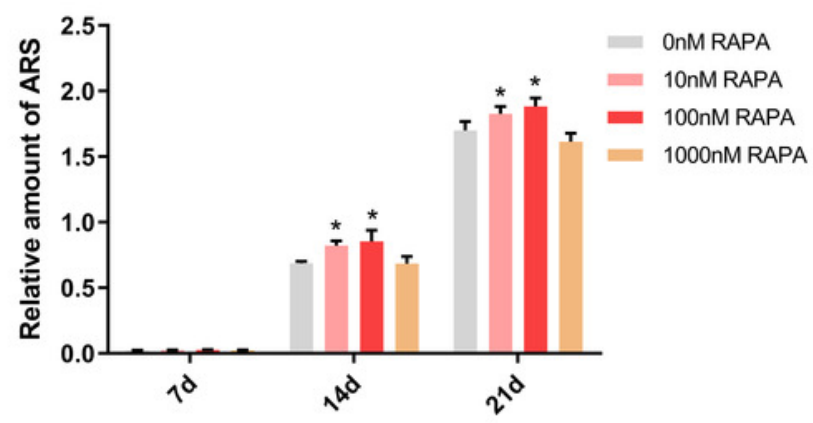

F

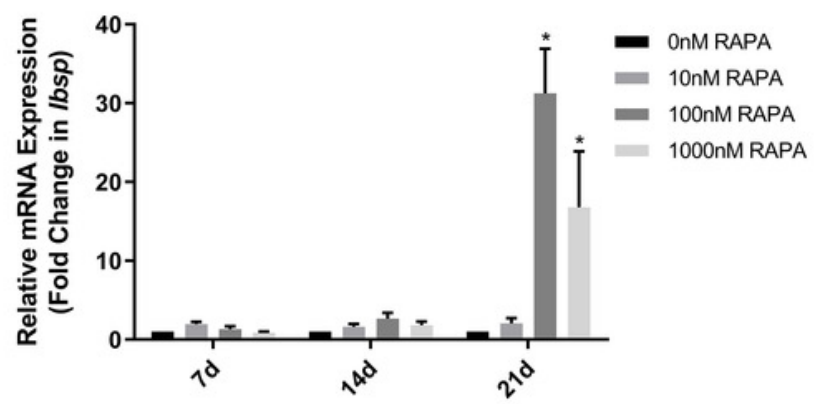

$\mathrm{H}$

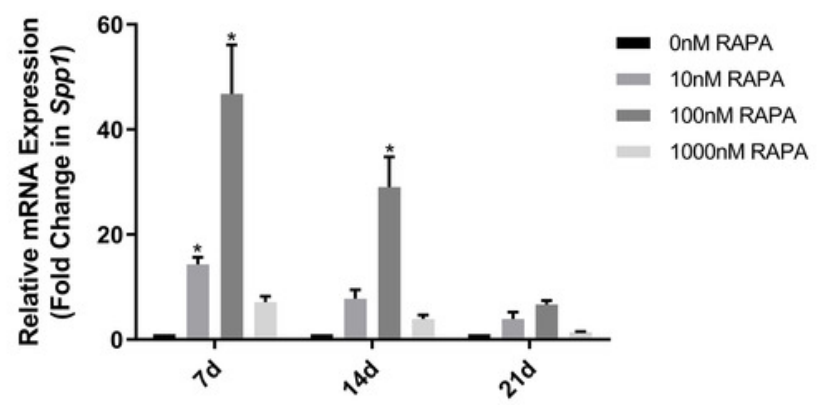




\section{Figure 4}

RAPA increased in vivo ectopic bone formation in MSMSCs.

Nude mice were implanted with MSMSCs treated with four concentrations of RAPA integrated with Bio-Oss ${ }^{\circledR}$. New bone-like structures (NB) with osteocyte-like cells (arrow) embedded within the calcified matrix was formed on the surface of Bio-Oss $®$ carrier. Connective tissue (CT) surrounded the carriers. (A) Histologic evaluation of H\&E-stained paraffin-embedded tissue sections. (B) Histologic evaluation of Masson's Trichrome-stained paraffin-embedded tissue sections. (C) Histologic evaluation of Goldner's Trichrome-stained paraffin-embedded tissue sections. Scale bars represent $50 \mu \mathrm{m}$. 


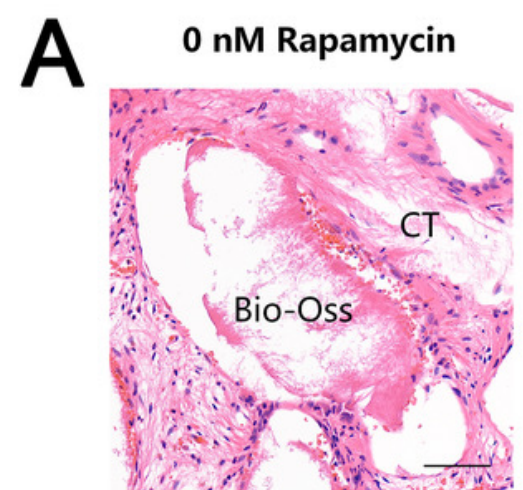

B
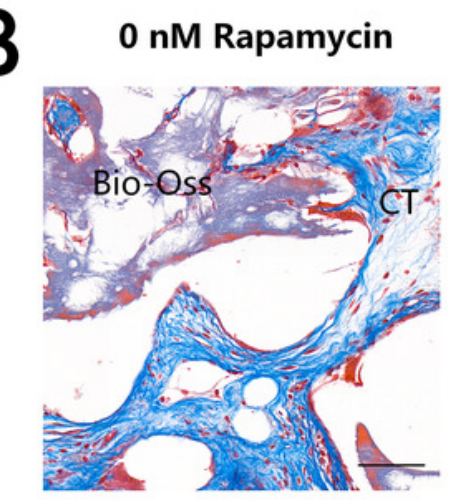

C

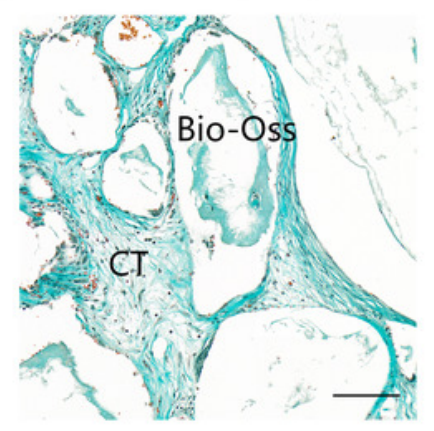

10 nM Rapamycin

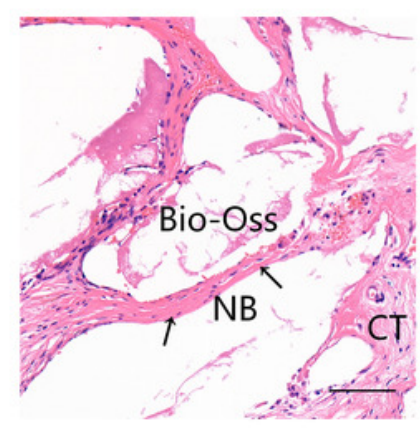

$10 \mathrm{nM}$ Rapamycin

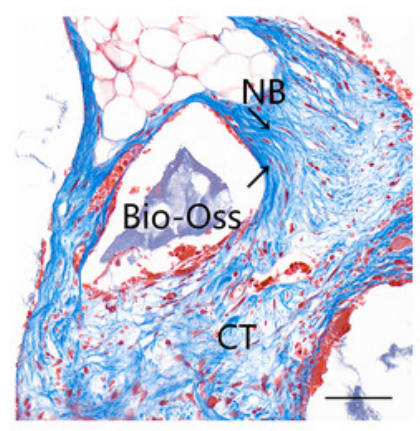

$10 \mathrm{nM}$ Rapamycin

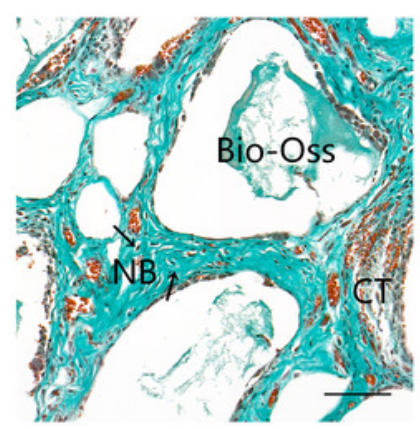

100 nM Rapamycin

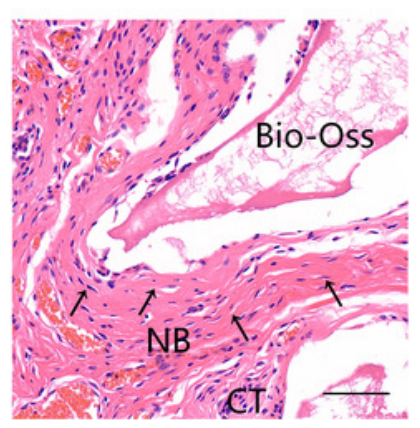

100 nM Rapamycin

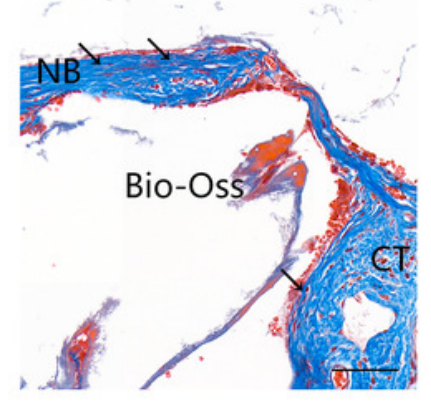

100 nM Rapamycin

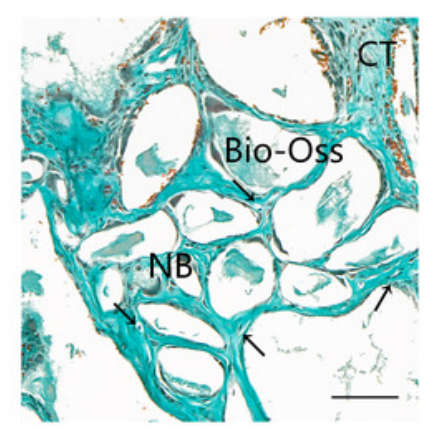

1000 nM Rapamycin

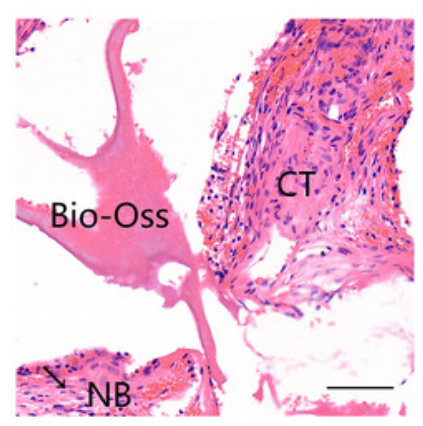

1000 nM Rapamycin

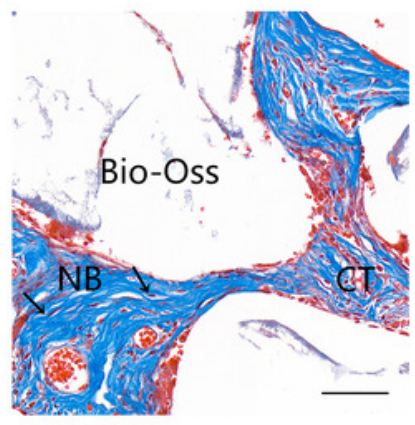

1000 nM Rapamycin

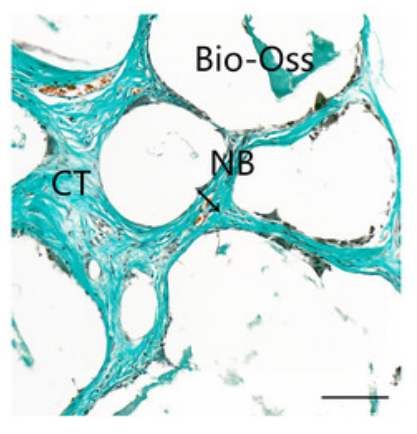

